To appear in Transportmetrica B: Transport Dynamics

Vol. 00, No. 00, Month 20XX, 1-22

\title{
A predictive continuum dynamic user-optimal model for a polycentric urban city
}

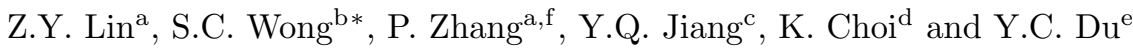 \\ ${ }^{a}$ Shanghai Institute of Applied Mathematics and Mechanics, Shanghai University, Shanghai, \\ China \\ ${ }^{b}$ Department of Civil Engineering, The University of Hong Kong, Pokfulam Road, Hong Kong \\ ${ }^{c}$ Department of Mathematics, Southwest University of Science and Technology, Mianyang, \\ Sichuan, China \\ ${ }^{d}$ Department of Transportation Engineering, TOD-based Sustainable Urban Transportation \\ Center, Ajou University, Republic of Korea \\ ${ }^{e}$ Key Lab. of Road and Traffic Engineering of the Ministry of Education, Tongji University, \\ Shanghai, China \\ ${ }^{f}$ Shanghai Key Laboratory of Mechanics in Energy Engineering \\ (Received 00 Month 20XX; final version received 00 Month 20XX)
}

\begin{abstract}
A predictive continuum dynamic user-optimal model is extended to investigate the traffic equilibrium problem for a polycentric urban city with multiple central business districts (CBDs). The road network within the city is assumed to be dense and can be viewed as a continuum in which travelers can choose their routes in a two-dimensional space. Travelers are assumed to choose their route to minimize the actual total cost to the destination (i.e., the CBD). The model consists of two parts: the conservation law part and the HamiltonJacobi part. The finite volume method is used to solve each part on unstructured meshes. Because the two parts are closely interconnected and have different initial times, solving the model can be treated as a fixed-point problem, which is solved using a self-adaptive method of successive averages. Numerical experiments for an urban city with two CBDs are presented to demonstrate the effectiveness of the model and the numerical algorithm.
\end{abstract}

Keywords: polycentric urban city; predictive user-optimal principle; elastic demand; unstructured meshes

\section{Introduction}

The traffic equilibrium problem is an important topic that has been considered and developed over recent decades. The literature devoted to the problem can be classified into two general approaches: the discrete modeling approach and the continuum modeling approach. The discrete modeling approach, in which the discrete road links constitute the network and each road link is modeled separately, is a conventional methodology used for the detailed analysis of a transportation system (Sheffi 1984). The continuum modeling approach focuses on the macroscopic characteristics of traffic flow in networks. It regards the road network as a continuum in which travelers can choose their routes in a two-dimensional space. The differences between two adjacent areas are relatively

\footnotetext{
*Corresponding author. E-mail: hhecwsc@hku.hk
} 


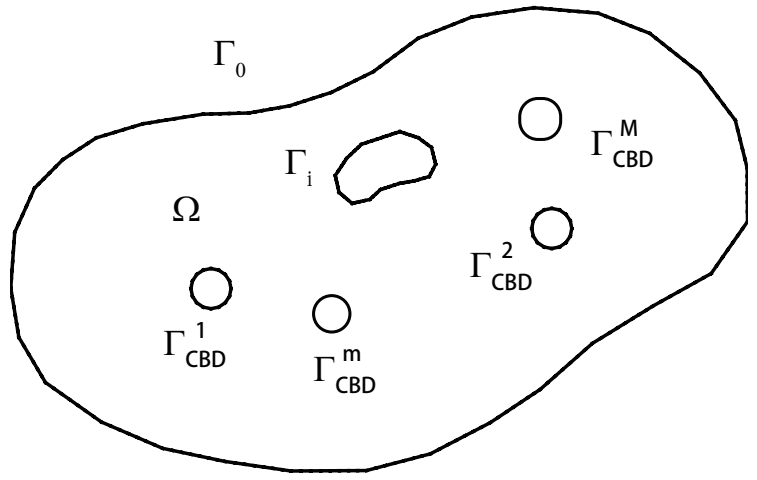

Figure 1. A polycentric urban city with an arbitrary configuration.

small compared with the variations over the entire network. Hence, the characteristic variables, such as the flow intensity, demand, density and travel cost, can be represented by smooth mathematical functions (Vaughan 1987; Wong and Wong 2015, 2016).

Most of the continuum modeling approaches for the traffic equilibrium problem are confined to the static case, in which the temporal variation of flow and cost are ignored, and hence cannot be used to study dynamic characteristics such as the travelers' departure/arrival time choices, evolution of the traffic congestion and dynamic traffic management and control (Blumenfeld 1977; Buckley 1979; Taguchi and Iri 1982; Wong 1998; Wong, Lee and Tong 1998; Ho and Wong 2007; Ho, Wong and Sumalee 2013; Yin et al. 2013; Du et al. 2016). To overcome these weaknesses, dynamic traffic assignment has received much attention in recent years. The route choice problem, which models travel behavior, is an important component of dynamic traffic assignment. Three major problems are considered in the route choice problem: the dynamic system-optimal problem, in which the total cost of the whole traffic system is minimized (Chow 2009; Nie 2011; Tao et al. 2014); the reactive dynamic user-optimal problem, in which the instantaneous total travel cost based on instantaneous information is minimized (Boyce, Ran and Leblanc 1995; Kuwahara and Akamatsu 2001; Hughes 2002; Jiang et al. 2009; Huang et al. 2009); and the predictive dynamic user-optimal (PDUO) problem, in which the actual total travel cost based on the assumption that travelers have perfect further information about the traffic conditions is minimized (Tong and Wong 2000; Lo and Szeto 2002; Hoogendoorn and Bovy 2004; Szeto and Lo 2004).

However, relatively few studies have investigated the continuum dynamic traffic assignment problem. Tao et al. (2014) presented a model for the dynamic system-optimal problem for a city in which the total cost of the system is minimized. Hughes (2002) provided a systematic framework for dynamic modeling of the pedestrian flow problem. Huang et al. (2009) showed that the route choice strategy in Hughes' model satisfies the reactive dynamic user-optimal principle and provided a numerical solution procedure. Jiang et al. (2014) developed a model for the reactive continuum dynamic user-optimal problem with elastic demand for a polycentric urban city. A numerical scheme based on unstructured (triangular) meshes is given to solve the model. For the predictive continuum dynamic user-optimal (PDUO-C) problem, Hoogendoorn and Bovy (2004) presented a model for pedestrians, in which the predictive dynamic user-optimal principle is satisfied. Although the models are presented for the pedestrian problem, they can also be 
applied to model the traffic flow in an urban city in a similar manner. Jiang et al. (2011) presented a model for the PDUO-C problem for an urban city with a single CBD, while Du et al. (2013) pointed out the inconsistency in the route-choice strategy under certain conditions, and constructed an improved route-choice strategy. In the work of Du et al., the urban city with one CBD is considered, and a numerical scheme based on rectangular meshes is developed. This paper extends the model into a polycentric urban city and has both theoretical and practical significance. Theoretically, we investigate multiclass traffic flow (corresponding to different CBDs) and prove that the extended model satisfies the predictive dynamic user equilibrium principle. In practical terms, cities with more than one CBD are common, and the presented model can be applied to investigate the traffic assignment problem in such a city. The other contribution of this paper is our construction of a numerical scheme based on unstructured (triangular) meshes. Our use of triangular meshes for spatial discretization facilitates handling of the regions complicated boundaries. Thus, the scheme is flexible for tracking arbitrary configurations and can also be used to simulate the numerical examples in Du et al. (2013), whereas the scheme based on rectangular meshes cannot simulate the numerical examples in this paper because of their complicated configurations.

The remainder of this paper is organized as follows. The description of the PDUO-C problem for a polycentric urban city is given in Section 2. Section 3 gives the model formulation, which includes the flow conservation law and the route-choice strategy. The solution algorithm is described in Section 4, and numerical experiments for a polycentric urban city with two CBDs based on unstructured meshes are presented in Section 5. Conclusions are made in Section 6.

\section{Problem description}

As shown in Figure 1, the considered polycentric urban city has $M(M \geq 2)$ compact CBDs. In the region $\Omega$, the road network is dense enough that it can be viewed as a continuum. The boundary of $\Omega$ is $\Gamma=\Gamma_{0} \cup \Gamma_{i} \cup \Gamma_{C B D}^{1} \cup \Gamma_{C B D}^{m} \cup \ldots \cup \Gamma_{C B D}^{M}$, where $\Gamma_{0}$ is the outer boundary, $\Gamma_{i}$ is the boundary of an obstruction such as a lake, park or some other area where traffic is not allowed to enter or leave, and $\Gamma_{C B D}^{m}(m=1, \ldots, M)$ is the boundary of the $m$-th CBD.

We assume that the travelers are classified into $M$ classes by their destinations (i.e., CBDs) and are distributed continuously in the region. The $m$-th class of traffic flow represents the travelers whose destination is the $m$-th CBD.

We denote the variables as follows. Under the condition of no confusion, the arbitrary function $f(x, y, t)$ can be written as $f$ for short.

- $\rho^{m}(x, y, t)\left(m=1, \ldots, M\right.$, in $\left.v e h / \mathrm{km}^{2}\right)$ is the density of the $m$-th class of traffic flow at location $(x, y)$ at time $t$.

- $\boldsymbol{v}^{m}=\left(u^{m}(x, y, t), v^{m}(x, y, t)\right)(m=1, \ldots, M)$ is the velocity vector of the $m$-th class of traffic flow at location $(x, y)$ at time $t . U^{m}(x, y, t)=\left|\boldsymbol{v}^{m}\right|($ in $k m / h)$ is the speed of the $m$-th class of traffic flow. It is determined by the sum of the densities at location $(x, y)$ at time $t$, i.e.,

$$
U^{m}(x, y, t)=U_{f}^{m}(x, y) e^{-\beta \rho^{2}(x, y, t)}, m=1, \ldots, M,
$$

where $U_{f}^{m}(x, y)$ (in $\left.k m / h r\right)$ is the free-flow speed of the $m$-th class of traffic flow at location $(x, y), \rho(x, y, t)=\sum_{m=1}^{M} \rho^{m}(x, y, t)$ and $\beta$ is a positive parameter reflecting the road condition.

- $\boldsymbol{F}^{m}=\left(f_{1}^{m}(x, y, t), f_{2}^{m}(x, y, t)\right)(m=1, \ldots, M)$ is the flow vector of the $m$-th class 
of traffic flow. The intensity of flow $\left|\boldsymbol{F}^{m}\right|$ (in veh/km/hr) is defined as

$$
\left|\boldsymbol{F}^{m}(x, y, t)\right|=\rho^{m}(x, y, t) U^{m}(x, y, t), m=1, \ldots, M .
$$

- $\phi^{m}(x, y, t)(m=1, \ldots, M$, in $\$)$ is the total actual travel cost of the $m$-th class of traffic flow from location $(x, y)$ to the $m$-th CBD.

- $q^{m}(x, y, t)\left(m=1, \ldots, M\right.$, in veh $\left./ \mathrm{km}^{2} / \mathrm{hr}\right)$ is the traffic elastic demand of the $m$-th class of traffic flow, which is associated with the total travel cost incurred by the $m$-th class of traffic flow and is represented by

$$
q^{m}(x, y, t)=q^{m}\left(\phi^{m}(x, y, t)\right) .
$$

Here, $q^{m}(x, y, t)$ is a monotonically decreasing function with $\phi^{m}(x, y, t)$.

- $c^{m}(x, y, t)(m=1, \ldots, M$, in $\$ / k m)$ is the cost distribution of the $m$-th class of traffic flow, which is defined as

$$
c^{m}(x, y, t)=\kappa\left(\frac{1}{U^{m}(x, y, t)}+\pi^{m}(\tilde{\rho}(x, y, t))\right),
$$

where $\tilde{\rho}(x, y, t)=\left\{\rho^{1}(x, y, t), \ldots, \rho^{M}(x, y, t)\right\}$, and $\kappa$ (in $\$ / \mathrm{hr}$ ) denotes the value of time. Compared with one CBD problem, the influence among different classes in polycentric problem should be considered. This connection is described by the cost distribution. The term $\frac{\kappa}{U^{m}(x, y, t)}$ represents the cost associated with the travel time, and $\kappa \pi^{m}(\tilde{\rho}(x, y, t))$ represents other related costs, such as the preference for avoiding conflict with the other class of traffic flow (for the different travel directions and destinations) and the high-density region.

\section{Model formulation}

In this section, we extend the PDUO-C model in ( $\mathrm{Du}$ et al. 2013) into a polycentric urban city with elastic traffic demand.

\subsection{Route-choice strategy}

Similar to the case in which the urban city has one CBD, the case of the city with multiple CBDs also has the following similar theorem. We state that "I|" means parallel and $\phi_{x}\left(\phi_{y}\right)$ means the partial differential of $\phi$ with respect to $x(y)$.

Theorem 1. If $\left(u^{m}, v^{m}\right) \|\left(-\phi_{x}^{m},-\phi_{y}^{m}\right)(\forall m=1, \ldots, M)$, then the predictive dynamic user-optimal principle is satisfied.

Proof. The proof is essentially the same as that in the case of one CBD: see Theorem 2 in (Du et al. 2013). We recall it as follows.

Note that $\phi^{m}(x, y, t)$ is the total travel cost of the $m$-th class of traffic flow incurred by a traveler who departs from location $(x, y)$ at time $t$ and travels to the city's CBD using the constructed route-choice strategy, namely, the route that satisfies $\left(u^{m}, v^{m}\right) \|\left(-\phi_{x}^{m},-\phi_{y}^{m}\right)$, and we denote this route as the "used" route. We need to prove that for any other "unused" route from location $O\left(x_{0}, y_{0}, t_{0}\right)$ that deviates from the "used" route, the total travel cost is not less than $\phi^{m}\left(x_{0}, y_{0}, t_{0}\right)$.

We first consider the "unused" route that deviates from the "used" route only at the initial $\Delta t$ time. We assume that $\Delta t$ is small enough such that the high-order terms in 
the Taylor expansion of $\phi^{m}(x, y, t)$ can be neglected. As shown in Figure 2, the circle with a radius $\Delta t U^{m}$ represents the traveler's locations after $\Delta t$ time from $O\left(x_{0}, y_{0}, t_{0}\right)$. $A(x, y)$ is the position at which the traveler arrives along the "used" route, where $\left(u^{m}, v^{m}\right) \|\left(-\phi_{x}^{m},-\phi_{y}^{m}\right)$ is the speed of the $m$-th class of traffic flow. $\tilde{A}(\tilde{x}, \tilde{y})$ is the position at which the traveler arrives along the route with the vector $\Delta t\left(u^{m}, v^{m}\right)$. We can obtain the change in $\phi^{m}$ along the "used" route:

$\phi^{m}\left(x, y, t_{0}+\Delta t\right)-\phi^{m}\left(x_{0}, y_{0}, t_{0}\right)=\left(\phi_{x}^{m}, \phi_{y}^{m}, \phi_{t}^{m}\right) \cdot\left(u^{m}, v^{m}, 1\right) \Delta t=\Delta t\left(-\left|\nabla \phi^{m}\right| U^{m}+\phi_{t}^{m}\right)$.

The change in $\phi^{m}$ along the "unused" route is

$\phi^{m}\left(\tilde{x}, \tilde{y}, t_{0}+\Delta t\right)-\phi^{m}\left(x_{0}, y_{0}, t_{0}\right)=\left(\phi_{x}^{m}, \phi_{y}^{m}, \phi_{t}^{m}\right) \cdot\left(\tilde{u}^{m}, \tilde{v}^{m}, 1\right) \Delta t \geq \Delta t\left(-\left|\nabla \phi^{m}\right| U^{m}+\phi_{t}^{m}\right)$.

Hence, we have

$$
\phi^{m}\left(\tilde{x}, \tilde{y}, t_{0}+\Delta t\right) \geq \phi^{m}\left(x, y, t_{0}+\Delta t\right) .
$$

Note that the travel costs from the location $O\left(x_{0}, y_{0}, t_{0}\right)$ to $A$ and $\tilde{A}$ are both $\Delta t U^{m} c^{m}\left(x_{0}, y_{0}, t_{0}\right)$. Therefore, we have

$$
\phi^{m}\left(x_{0}, y_{0}, t_{0}\right)=\Delta t U^{m} c^{m}\left(x_{0}, y_{0}, t_{0}\right)+\phi^{m}\left(x, y, t_{0}+\Delta t\right)
$$

and the total travel cost along the "unused" route is

$$
\tilde{\phi}^{m}=\Delta t U^{m} c^{m}\left(x_{0}, y_{0}, t_{0}\right)+\phi^{m}\left(\tilde{x}, \tilde{y}, t_{0}+\Delta t\right) .
$$

So, the total cost along the "unused" route is no less than $\phi^{m}\left(x_{0}, y_{0}, t_{0}\right)$.

If the traveler continues to move in a direction deviating from the "used" route after the initial $\Delta t$ time, he/she will arrive at the $m$-th CBD with a total cost $\tilde{\tilde{\phi}}^{m} \geq \tilde{\phi}^{m} \geq$ $\phi^{m}\left(x_{0}, y_{0}, t_{0}\right)$. Therefore, for any "unused" route, the total cost must be no less than $\phi^{m}\left(x_{0}, y_{0}, t_{0}\right)$.

The condition $\left(u^{m}, v^{m}\right) \|\left(-\phi_{x}^{m},-\phi_{y}^{m}\right)$ in Theorem 1 means that travelers choose to move in the direction of the descending maximum cost. In other words, the travelers move along the dynamic routes of the minimum predicted actual costs. If the condition is satisfied, the traffic reaches the predictive dynamic user-optimal state. Next, we prove that $\left(u^{m}, v^{m}\right) \|\left(-\phi_{x}^{m},-\phi_{y}^{m}\right)$ is a necessary and sufficient condition for the HamiltonJacobi formulation $\frac{1}{U^{m}} \phi_{t}^{m}-\left|\nabla \phi^{m}\right|=-c^{m}$, where $\nabla \phi^{m}=\left(\phi_{x}^{m}, \phi_{y}^{m}\right)$. Then, based on Theorem 1, if the Hamilton-Jacobi formulation is satisfied, the predictive dynamic user-optimal principle is satisfied.

We define a route along a given speed vector of the $m$-th class of traffic flow as $(x(t), y(t))$ with parameter $t$. Along this route, we have

$$
\frac{d \phi^{m}}{d t}=\phi_{x}^{m} \frac{d x(t)}{d t}+\phi_{y}^{m} \frac{d y(t)}{d t}+\phi_{t}^{m}=\phi_{x}^{m} u^{m}+\phi_{y}^{m} v^{m}+\phi_{t}^{m}, \quad m=1, \ldots, M
$$




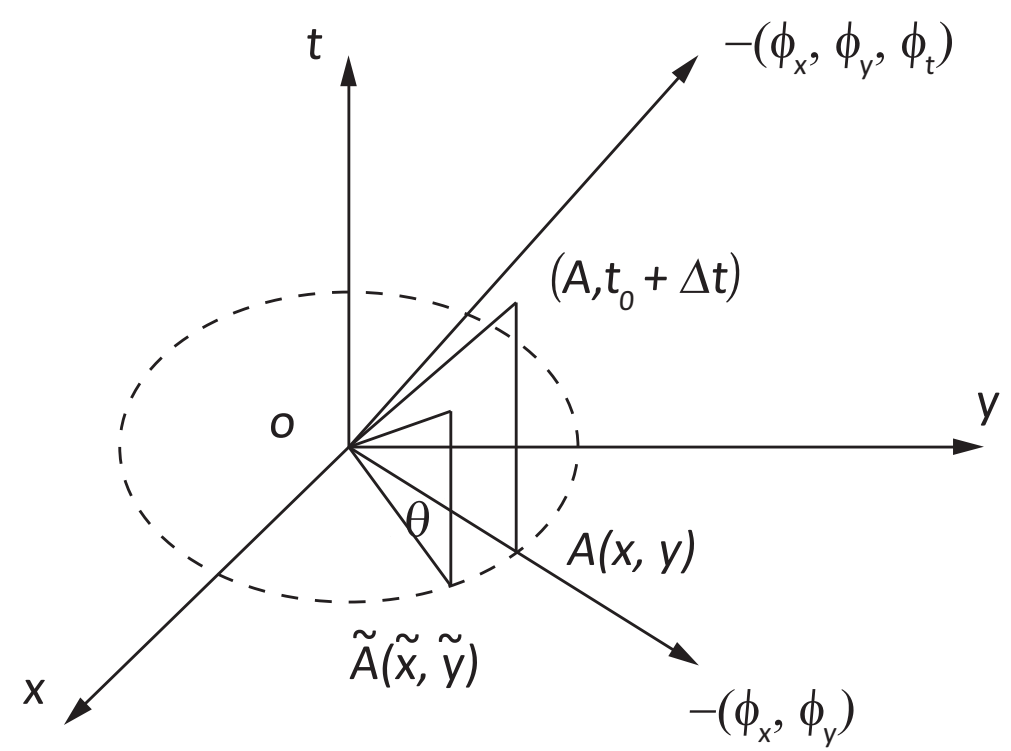

Figure 2. The "used" and "unused" routes.

By the definition, we also have

$$
\begin{aligned}
\frac{d \phi^{m}}{d t} & =\lim _{\Delta t \rightarrow 0} \frac{\phi^{m}(x(t+\Delta t), y(t+\Delta t), t+\Delta t)-\phi^{m}(x(t), y(t), t)}{\Delta t} \\
& =-U^{m}(x(t), y(t), t) \lim _{\Delta t \rightarrow 0} \frac{\phi^{m}(x(t), y(t), t)-\phi^{m}(x(t+\Delta t), y(t+\Delta t), t+\Delta t)}{\Delta t U^{m}(x(t), y(t), t)} \\
& =-U^{m}(x, y, t) c^{m}(x, y, t), \quad m=1, \ldots, M .
\end{aligned}
$$

If

$$
\left(u^{m}, v^{m}\right) \|\left(-\phi_{x}^{m},-\phi_{y}^{m}\right), \quad m=1, \ldots, M,
$$

by considering Eqs.(3) and (4), we have the Hamilton-Jacobi formulation

$$
\frac{1}{U^{m}} \phi_{t}^{m}-\left|\nabla \phi^{m}\right|=-c^{m}, m=1, \ldots, M
$$

If the Hamilton-Jacobi equation (5) is satisfied, we also have

$$
\phi_{x}^{m} u^{m}+\phi_{y}^{m} v^{m}+\phi_{t}^{m}=-\left|\nabla \phi^{m}\right| U^{m}+\phi_{t}^{m}, m=1, \ldots, M .
$$

This means that

$$
\left(u^{m}, v^{m}\right) \|\left(-\phi_{x}^{m},-\phi_{y}^{m}\right), m=1, \ldots, M,
$$

and therefore the predictive dynamic user-optimal principle is satisfied.

\subsection{The complete model}

We regard the classes of traffic flow as different compressible continuum fluids and each class distribute continuously in the whole region. Similar to mass conservation in fluid 
mechanics, for each class of traffic flow, the density, flow, and demand satisfy the following conservation law:

$$
\rho_{t}^{m}(x, y, t)+\nabla \cdot \boldsymbol{F}^{m}(x, y, t)=q^{m}(x, y, t), \quad m=1, \ldots, M .
$$

This means that, for a small area and for each class of traffic flow, the increase in the number of vehicles $\left(\rho^{m}\right)$ equals the sum of newly generated vehicles $\left(q^{m}\right)$ and vehicles moving into the area, less those moving out $\left(-\nabla \cdot \boldsymbol{F}^{m}\right)$. To satisfy the predictive dynamic user-optimal principle, we choose the velocity vector $\boldsymbol{v}^{m}=\left(u^{m}, v^{m}\right) \|-\left(\phi_{x}^{m}, \phi_{y}^{m}\right)(m=$ $1, \ldots, M)$. Hence, we have

$$
\boldsymbol{F}^{m}=\rho^{m} \boldsymbol{v}^{m}=-\rho^{m} U^{m} \frac{\nabla \phi^{m}}{\left|\nabla \phi^{m}\right|}, \quad m=1, \ldots, M .
$$

Now, we divide the complete model into two parts.

\section{The conservation law part is}

$$
\left\{\begin{array}{lrr}
\rho_{t}^{m}+\nabla \cdot \boldsymbol{F}^{m}=q^{m}, & \forall(x, y) \in \Omega, \quad t \in T, & \\
\boldsymbol{F}^{m}=-\rho^{m} U^{m} \frac{\nabla \phi^{m}}{\left|\nabla \phi^{m}\right|}, & \forall(x, y) \in \Omega, \quad t \in T, & \forall m=1, \ldots, M . \\
\boldsymbol{F}^{m}(x, y, t) \cdot \boldsymbol{n}=0, & \forall(x, y) \in \Gamma \backslash \Gamma_{C B D}^{m}, & t \in T, \\
\rho^{m}(x, y, 0)=\rho_{0}^{m}(x, y), & \forall(x, y) \in \Omega,
\end{array}\right.
$$

Here, $\rho_{0}^{m}(x, y)$ is the initial density of the $m$-th class of traffic flow. We assume that no vehicle is allowed to enter the obstruction of the other CBD or leave the urban city. Thus we have the boundary condition $\boldsymbol{F}^{m}(x, y, t) \cdot \boldsymbol{n}=0, \forall(x, y) \in \Gamma \backslash \Gamma_{C B D}^{m}, t \in T$.

The Hamilton-Jacobi part is

$$
\left\{\begin{array}{lrr}
\frac{1}{U^{m}} \phi_{t}^{m}-\left|\nabla \phi^{m}\right|=-c^{m}, & \forall(x, y) \in \Omega, \quad t \in T, & \\
\phi^{m}(x, y, t)=\phi_{C B D}^{m}, & \forall(x, y) \in \Gamma_{C B D}^{m}, & t \in T, \\
\phi^{m}\left(x, y, t_{\text {end }}\right)=\phi_{0}^{m}(x, y), & \forall(x, y) \in \Omega, &
\end{array}\right.
$$

where the initial condition of $\phi_{0}^{m}(x, y)(m=1, \ldots, M)$ is given by the Eikonal equation:

$$
\left\{\begin{array}{lr}
\left|\nabla \phi_{0}^{m}(x, y)\right|=c^{m}\left(x, y, t_{\text {end }}\right), & \forall(x, y) \in \Omega, \\
\phi_{0}^{m}(x, y)=\phi_{C B D}^{m}, & \forall(x, y) \in \Gamma_{C B D}^{m} .
\end{array}\right.
$$

The initial time is set at $t=t_{\text {end }}$ because the travel cost to a CBD only depends on the situations in the future. The Eikonal equation can be solved by using the fast marching method (see Huang et al. (2009) and Jiang et al. (2009) for details). The density $\rho^{m}(x, y, t)(m=1, \ldots, M)$ is governed by the conservation law, and total travel cost $\phi^{m}(x, y, t)(m=1, \ldots, M)$ is governed by the Hamilton-Jacobi equation. When computing the density, we must know the total cost, and when computing the cost, we also need to know the density. Noting the different initial times of the two parts, we consider the solution procedure as a fixed-point problem and use a self-adaptive method of successive averages (MSA) to solve it. The self-adaptive MSA is proposed in (Du et al. 2013) to solve the fixed-point problem in the PDUO-C model, which can automatically determine the optimal size, and thus improve the convergence rate. 


\section{Solution algorithm}

In Section 3, the complete model is divided into two parts: the conservation law part and the Hamilton-Jacobi part. In this section, we present the solution algorithm on the unstructured meshes. We use the finite volume method to solve both the conservation equations and the Hamilton-Jacobi equations and a self-adaptive MSA to solve the fixedpoint problem.

The unstructured meshes are used to handle the complicated configuration of the urban city. We divide the region $\Omega$ with triangular meshes. Let $X_{i}\left(i=1, \ldots, N_{P}\right)$, $T_{i}\left(i=1, \ldots, N_{T}\right)$ be a node and a triangle, respectively, within the domain. $N_{T}, N_{P}$ are the number of triangles and nodes, respectively. As shown in Figure $3, A_{i}, N_{i k}$ and $l_{i k}$ are the area, the $k$-th node and $k$-th side of $T_{i}$, respectively. $n_{i k}$ is the unit outer vector through the side $l_{i k}$. The $k$-th neighboring triangle of $T_{i}$ is denoted by $T_{i k}$.

\subsection{Finite volume method used to solve the conservation equations and the Hamilton-Jacobi equations}

We first consider the finite volume method to compute $\rho^{m}(x, y, t)(m=1, \ldots, M)$ from the conservation equation. Here, we assume that the total travel cost $\phi^{m}(x, y, t)(m=$ $1, \ldots, M)$ of each class of traffic flow is known. The triangular cell $T_{i}\left(i=1, \ldots, N_{T}\right)$ represents a control volume, and the density is stored in the geometric center.

Integrating Eq. (6) and using the Gauss theorem, we have

$$
\frac{\partial \rho^{m}\left(x_{i}, y_{i}, t\right)}{\partial t}+\frac{1}{A_{i}} \oint_{\partial T_{i}} \boldsymbol{F}^{m}(x, y, t) \cdot \boldsymbol{n}_{\boldsymbol{i}} d s=q^{m}\left(\phi^{m}\left(x_{i}, y_{i}, t\right), t\right), m=1, \ldots, M,
$$

where $\left(x_{i}, y_{i}\right), \partial T_{i}$ are the geometric center and boundary of cell $T_{i}$ respectively, and $\boldsymbol{n}_{i}$ is the outward unit vector through $\partial T_{i}$. The integral average of $q^{m}$ in $T_{i}$ is approximated as $q^{m}\left(x_{i}, y_{i}, t\right)$. The item $\oint_{\partial T_{i}} \boldsymbol{F}^{m}(x, y, t) \cdot \boldsymbol{n}_{\boldsymbol{i}} d s$ is approximated by

$$
\oint_{\partial T_{i}} \boldsymbol{F}^{m} \cdot \boldsymbol{n}_{\boldsymbol{i}} d s=\sum_{k=1}^{3} \tilde{\boldsymbol{F}}_{i k}^{m} \cdot \boldsymbol{n}_{i k}\left|l_{i k}\right|
$$

where $\tilde{\boldsymbol{F}}_{i k}^{m}$ is the numerical flux through the $k$-th surface $l_{i k}$ with length of $\left|l_{i k}\right|$. The Lax-Friedrichs numerical flux is adopted, i.e.,

$$
\tilde{\boldsymbol{F}}_{i k}^{m} \cdot \boldsymbol{n}_{i k}=\frac{1}{2}\left[\boldsymbol{F}\left(\rho_{i k}^{m, l}\right) \cdot \boldsymbol{n}_{i k}+\boldsymbol{F}\left(\rho_{i k}^{m, r}\right) \cdot \boldsymbol{n}_{i k}-c\left(\rho_{i k}^{m, r}-\rho_{i k}^{m, l}\right)\right], \quad k=1,2,3,
$$

where $\rho_{i k}^{m, r}, \rho_{i k}^{m, l}$ are the values at the right and left of side $l_{i k}$, and

$$
c=\max _{i, k}\left|\boldsymbol{F}^{m \prime}\left(\rho^{m}\right) \cdot \boldsymbol{n}\right|_{i, k}
$$

See (Jiang et al. 2009; Jiang et al. 2011) for details of the numerical flux.

For the time discretization, the first-order Euler scheme is used, and the density at the $n+1$ time step can be obtained as

$$
\left(\rho^{m}\right)_{i}^{n+1}=\left(\rho^{m}\right)_{i}^{n}-\frac{\Delta t}{A_{i}} \sum_{k=1}^{3} \tilde{\boldsymbol{F}}_{i k}^{m} \cdot \boldsymbol{n}_{i k}\left|l_{i k}\right|+\Delta t\left(q^{m}\right)_{i}^{n},
$$


where the time step $\Delta t$ must satisfy the Courant-Friedrichs-Lewy condition

$$
\Delta t \leq \min _{i=1, \ldots, N_{T}, k=1,2,3} \frac{A_{i}}{\left|l_{i k}\right|\left|\boldsymbol{F}^{m^{\prime}}\left(\rho^{m}\right) \cdot \boldsymbol{n}\right|} .
$$

For the time-dependent Hamilton-Jacobi equation, the value of $\phi^{m}(m=1, \ldots, M)$ at node $X_{i}$ is approximated by $\phi_{i}^{m} . \nabla \phi^{m}(m=1, \ldots, M)$ at $T_{i k}$ is denoted by $\left(\nabla \phi^{m}\right)_{i k}$. We assume the density $\rho^{m}(x, y, t)(m=1, \ldots, M)$ is known. Noting that the initial time of the Hamilton-Jacobi equation is $t=t_{\text {end }}$, we define

$$
\tau=t_{\text {end }}-t, \quad \Phi^{m}(x, y, \tau)=\phi^{m}\left(x, y, t_{\text {end }}-\tau\right), \quad m=1, \ldots, M
$$

and thus we can rewrite Eqs. (8) in the following form:

$$
\left\{\begin{array}{lrr}
\frac{1}{U^{m}} \Phi_{\tau}^{m}+\left|\nabla \Phi^{m}\right|=c^{m}, & \forall(x, y) \in \Omega, & \tau \in T, \\
\Phi^{m}(x, y, \tau)=\phi_{C B D}^{m}, & \forall(x, y) \in \Gamma_{C B D}^{m}, & \tau \in T, \\
\Phi^{m}(x, y, 0)=\phi_{0}^{m}(x, y), & \forall(x, y) \in \Omega, &
\end{array}\right.
$$

We define

$$
H\left(\nabla \Phi^{m}\right)=U^{m}\left(\left|\nabla \Phi^{m}\right|-c^{m}\right),
$$

and then the scheme to solve equation $\Phi_{\tau}^{m}+H\left(\nabla \Phi^{m}\right)=0$ is

$$
\left(\Phi^{m}\right)_{i}^{n+1}=\left(\Phi^{m}\right)_{i}^{n}-\Delta t \hat{H}\left(\left(\nabla \Phi^{m}\right)_{i 1}, \ldots,\left(\nabla \Phi^{m}\right)_{i I_{i}}\right),
$$

where $I_{i}$ is the number of the elements around node $X_{i}$.

We assume that $\Phi^{m}(x, y, \tau)$ on each element $T_{i k}$ is approximated by a linear function with the values of $\Phi^{m}$ at three vertices. Thus, its gradient $\left(\nabla \Phi^{m}\right)_{i k}$ on $T_{i k}$ is approximated by a constant vector. The global Lax-Friedrichs numerical flux is adopted (Abgrall 1996):

$\hat{H}\left(\left(\nabla \Phi^{m}\right)_{i 1}, \ldots,\left(\nabla \Phi^{m}\right)_{i I_{i}}\right)=H\left(\frac{\sum_{k=1}^{I_{i}}\left(\nabla \Phi^{m}\right)_{i k}}{2 \pi}\right)-\frac{\alpha}{\pi} \sum_{k=1}^{I_{i}} \beta_{i k+\frac{1}{2}}\left(\frac{\left(\nabla \Phi^{m}\right)_{i k}+\left(\nabla \Phi^{m}\right)_{i k+1}}{2}\right) \cdot \boldsymbol{n}_{i k+\frac{1}{2}}$,

where

$$
\begin{gathered}
\beta_{i k+\frac{1}{2}}=\tan \frac{\theta_{i k}}{2}+\tan \frac{\theta_{i k+1}}{2}, \\
\alpha=\max \left\{\max _{A \leq a \leq B, C \leq b \leq D}\left|H_{2}(a, b)\right|, \max _{A \leq a \leq B, C \leq b \leq D}\left|H_{1}(a, b)\right|\right\} .
\end{gathered}
$$

Here, $H_{1}$ and $H_{2}$ are the partial derivatives of $H$ with $a$ and $b$, respectively. $[A, B],[C, D]$ are the value range of $\Phi_{x}^{m}$ and $\Phi_{y}^{m}$ respectively. $\theta_{i k}$ is the angle of the $T_{i k}$ at node $X_{i}$, and $\boldsymbol{n}_{i k+\frac{1}{2}}$ is the unit vector along the common side of $T_{i k}$ and $T_{i k+1}$ (see Figure 3 ). The time step $\Delta t$ must satisfy the Courant-Friedrichs-Lewy condition

$$
\Delta t \leq \frac{h}{2 \alpha},
$$




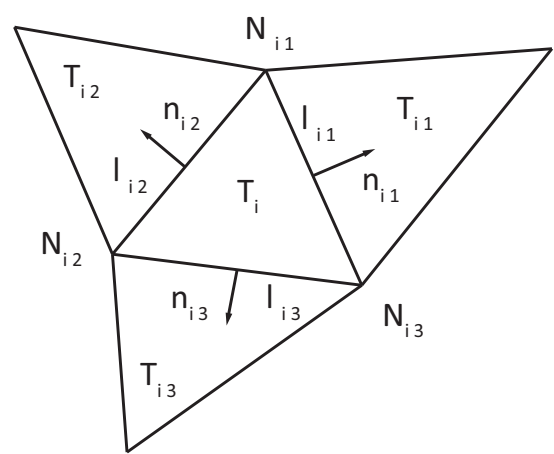

(a) Element

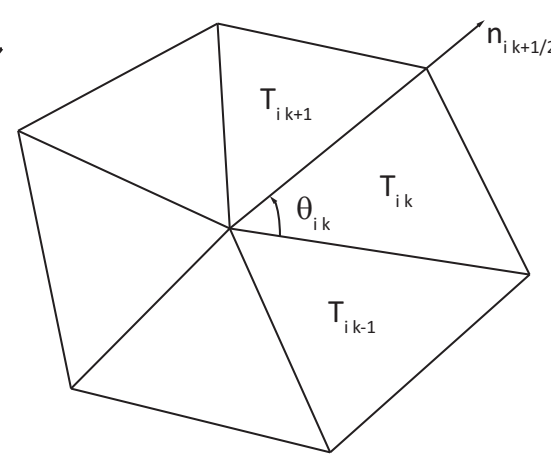

(b) Node

Figure 3. The unstructured meshes.

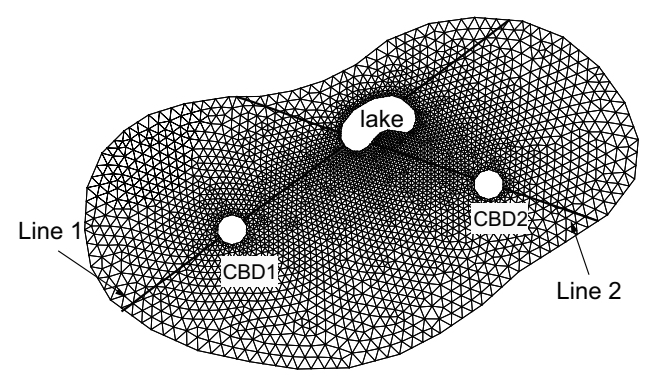

Figure 4. A polycentric urban city with two CBDs.

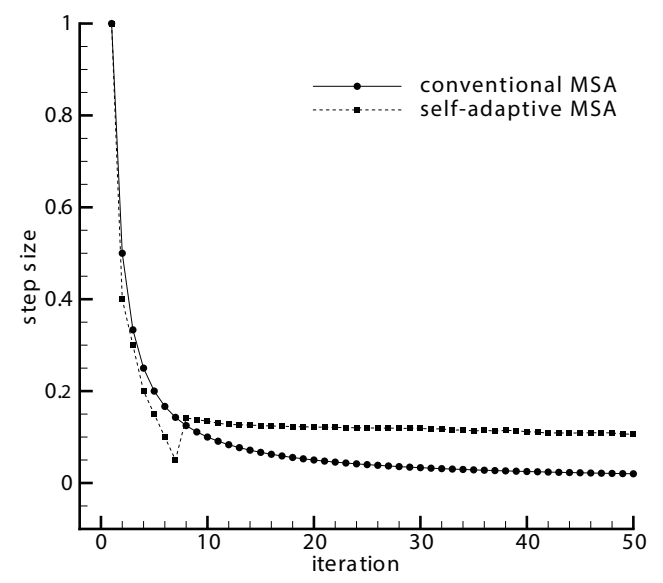

Figure 5. The step sizes of the self-adaptive MSA and the conventional MSA.

where $h$ is the smallest radius of the circles of center $X_{i}$ and contained in $\bigcup_{k} T_{i k}, i=$ $1, \ldots, N_{P}$.

\subsection{Fixed-point problem and a self-adaptive $M S A$}

Noting that the model's two parts are interconnected and the initial times are different, we consider it as a fixed-point problem and solve it by using a self-adaptive MSA. 


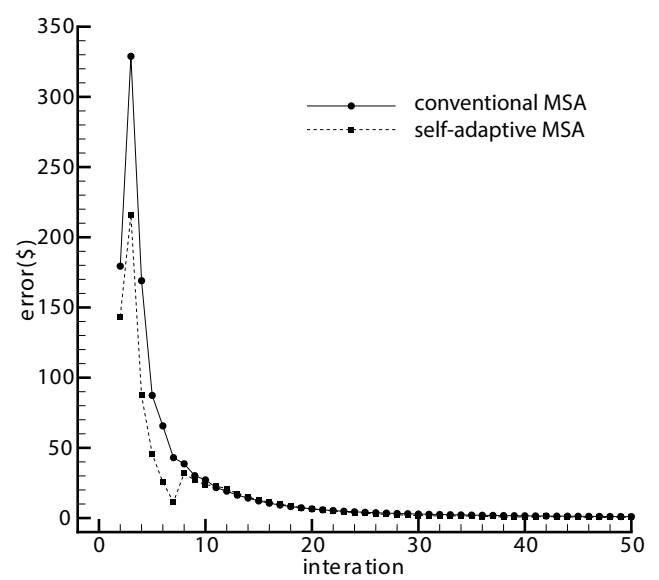

Figure 6. The errors of the self-adaptive MSA and the conventional MSA.

Define the vectors of the numerical solutions as

$$
\begin{aligned}
& \vec{\rho}=\left\{\rho_{i}^{m, n}, m=1, \ldots, M, i=1, \ldots, N_{T}, n=1, \ldots, N_{t}\right\}, \\
& \vec{\phi}=\left\{\phi_{i}^{m, n}, m=1, \ldots, M, i=1, \ldots, N_{T}, n=1, \ldots, N_{t}\right\},
\end{aligned}
$$

where $N_{t}$ is the number of time steps.

We define one iteration of the MSA as following two steps.

Step 1. With a known vector $\vec{\phi}^{\text {old }}$, we solve the conservation law part Eq. (7) from $t=0$ to $t=t_{\text {end }}$ using the finite volume method, and thus we have

$$
\vec{\rho}=\boldsymbol{g}\left(\vec{\phi}^{\text {old }}\right)
$$

Step 2. We solve the Hamilton-Jacobi part Eq. (8) from $t=t_{\text {end }}$ to $t=0$ using the finite volume method, and thus we have

$$
\vec{\phi}^{n e w}=\boldsymbol{h}(\vec{\rho}) .
$$

We denote the iteration by

$$
\vec{\phi}^{\text {new }}=\boldsymbol{h}\left(\boldsymbol{g}\left(\vec{\phi}^{o l d}\right)\right)=\boldsymbol{f}\left(\vec{\phi}^{\text {old }}\right) .
$$

Next, we solve the fixed-point problem

$$
\vec{\phi}=\boldsymbol{f}(\vec{\phi})
$$

using a self-adaptive MSA.

We denote the solution before the $k$-th iteration by $\vec{\phi}^{k}$ and compute $\vec{\phi}^{k+1}$ with following steps.

Step 1. We solve the solution $\vec{y}^{k}=\boldsymbol{f}\left(\vec{\phi}^{k}\right)$ in the $k$-th iteration. 


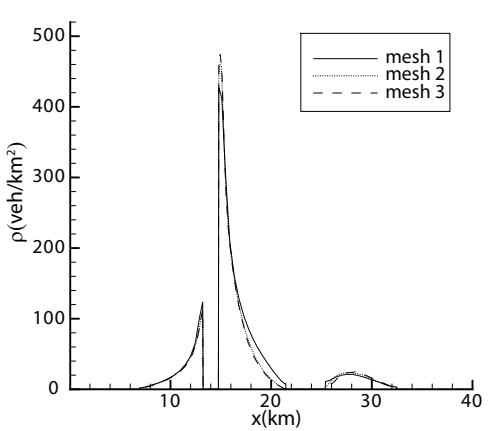

(a) $t=1 \mathrm{hr}$

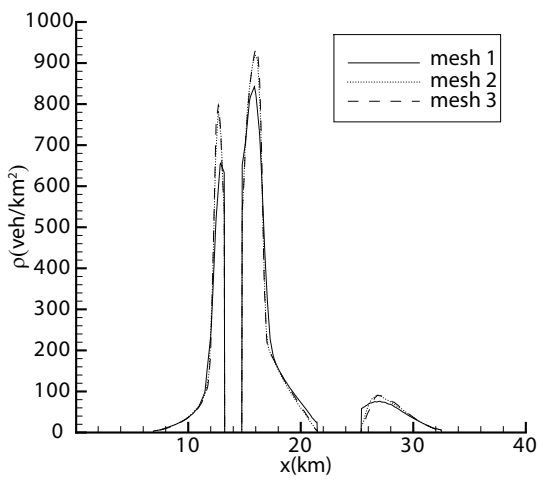

(c) $t=2 \mathrm{hr}$

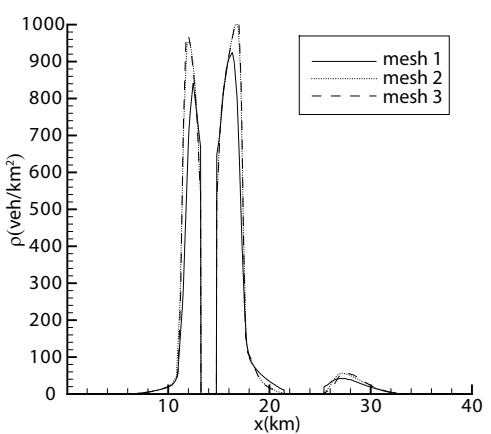

(e) $t=3 \mathrm{hr}$

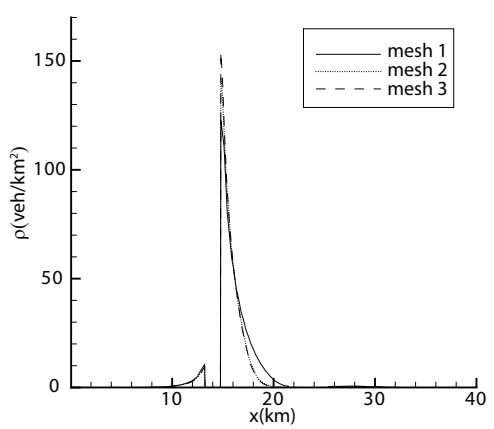

(g) $t=4 h r$

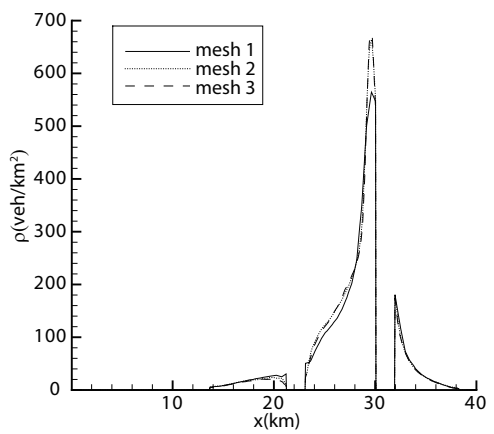

(b) $t=1 \mathrm{hr}$

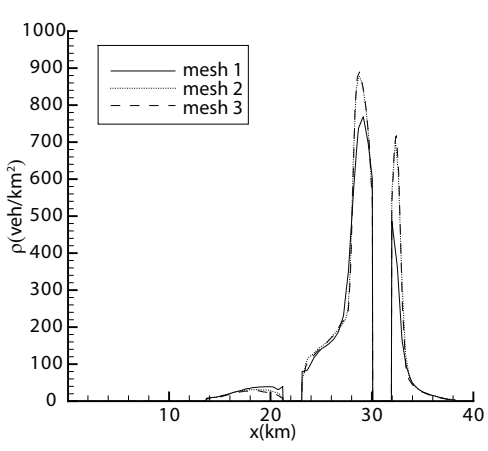

(d) $t=2 h r$

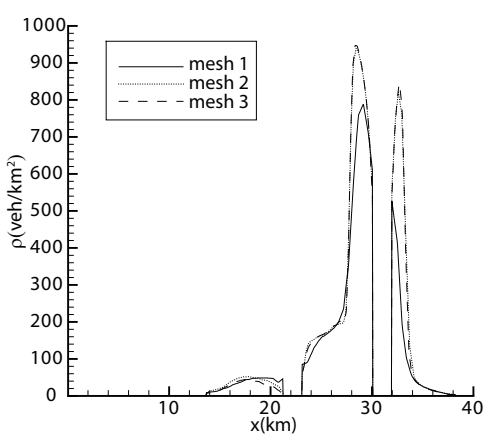

(f) $t=3 \mathrm{hr}$

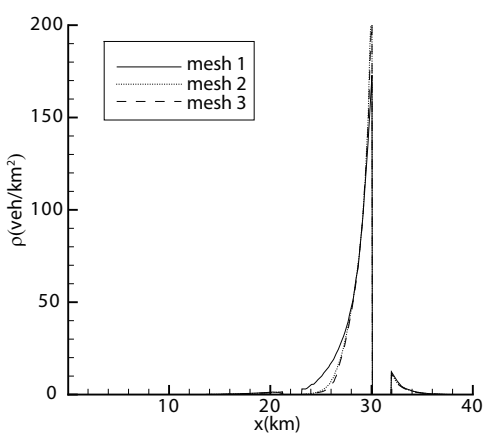

(h) $t=4 \mathrm{hr}$

Figure 7. Convergence curves (left: density of Class 1 along Line 1; right: density of Class 2 along Line 2). 
Step 2. Choosing a step size $\lambda_{k}$, we have

$$
\vec{\phi}^{k+1}=\left(1-\lambda_{k}\right) \vec{\phi}^{k}+\lambda_{k} \cdot \vec{y}^{k} .
$$

The convergence is reached if

$$
\left\|\vec{\phi}^{k+1}-\vec{\phi}^{k}\right\| \leq \delta
$$

where $\delta$ is the convergence threshold value and is set as 0.01 .

The choice of the step size is important to the convergence speed of the MSA. A predetermined step size $\lambda_{k}=1 / k$ was suggested by Robbins and Monro (1951). However, the step sizes become too small after a number of iterations, such that the convergence speed is very slow. Du et al. (2013) proposed a self-adaptive MSA to automatically determine the optimal step size by using the least squares approach. We rewrite it as follows.

1. For the first several iterations, the step sizes are set as:

$$
\lambda_{1}=1.0, \lambda_{2}=0.4, \lambda_{3}=0.3, \lambda_{4}=0.2, \lambda_{5}=0.15, \lambda_{6}=0.1, \lambda_{7}=0.05
$$

2. For $k>2$, we record the step size $\lambda_{k-1}$ and compute the ratio of error

$$
r_{k-1}=\frac{\left\|\vec{\phi}^{k}-\boldsymbol{f}\left(\vec{\phi}^{k}\right)\right\|^{2}}{\left\|\vec{\phi}^{k-1}-\boldsymbol{f}\left(\vec{\phi}^{k-1}\right)\right\|^{2}},
$$

and thus we use the points $\left(\lambda_{k-1}, r_{k-1}\right)$ to fit the curve $r^{*}(\lambda)$.

3. For the $n+1(n \geq 7)$ step, we use the least squares method to fit the points $\left(\lambda_{k}, r_{k}\right), k=2, \ldots, n$. Here, we require the fitted curve to pass through the point $(0,1)$. Determine the step size $\lambda_{n+1}$ as the minimum point of the fitted curve $r^{*}(\lambda)$, i.e., $r^{*}\left(\lambda_{n+1}\right)=\min _{\lambda} r^{*}(\lambda)$. If $\lambda_{n+1} \leq 0$ or $\lambda_{n+1} \geq 1$, we set $\lambda_{n+1}=0.5 \lambda_{n}$.

\subsection{Solution procedure}

To start the self-adaptive MSA, the first iteration should be given because we have no information on the actual total cost at the initial time. We compute the density from 0 to $t_{\text {end }}$ by solving the following reactive dynamic user-optimal model (Jiang et al. 2014, 2009; Huang et al. 2009):

$$
\begin{cases}\rho_{t}^{m}+\nabla \cdot \boldsymbol{F}^{m}=q^{m}, & \forall(x, y) \in \Omega, \quad t \in T, \\ \boldsymbol{F}^{m}=-\rho^{m} U^{m} \frac{\nabla \phi^{m}}{\left|\nabla \phi^{m}\right|}, & \forall(x, y) \in \Omega, \quad t \in T, \quad \forall m=1, \ldots, M, \\ \left|\nabla \phi^{m}\right|=c^{m}(x, y, t), & \forall(x, y) \in \Omega, \quad t \in T,\end{cases}
$$

and the initial boundary conditions are

$$
\left\{\begin{array}{rrr}
\boldsymbol{F}^{m}(x, y, t) \cdot \boldsymbol{n}=0, & \forall(x, y) \in \Gamma \backslash \Gamma_{C B D}^{m}, \quad t \in T, \\
\rho^{m}(x, y, 0)=\rho_{0}^{m}(x, y), & \forall(x, y) \in \Omega, \\
\phi^{m}(x, y, t)=\phi_{C B D}^{m}, & \forall(x, y) \in \Gamma_{C B D}^{m}, \quad t \in T, \ldots, M .
\end{array}\right.
$$


In this model, the instantaneous total cost is computed at each time step, and the routechoice strategy that satisfies the reactive dynamic user-optimal principle is used. We can consider the instantaneous total cost as $\vec{\phi}^{1}$.

We write the solution procedure as follows.

1. Compute the density $\boldsymbol{\rho}^{1}$ using Eqs. (11). Compute the total cost

$$
\vec{y}^{1}=\boldsymbol{h}\left(\vec{\rho}^{1}\right)
$$

by solving Eqs. (9) and obtain $\vec{\phi}^{2}=\left(1-\lambda_{1}\right) \vec{\phi}^{1}+\lambda_{1} \vec{y}^{1}$.

2. For $k \geq 2$, using the $k$-th numerical solution $\vec{\phi}^{k}$, we have

$$
\vec{y}^{k}=\boldsymbol{h}\left(\boldsymbol{g}\left(\vec{\phi}^{k}\right)\right)=\boldsymbol{f}\left(\vec{\phi}^{k}\right) .
$$

3. Compute the step sizes $\lambda_{k}(k>7)$ using the method described in Section 4.2. The $\lambda_{k}(k=1, \ldots, 7)$ are predetermined.

4. Compute the $(k+1)$-th solution vector

$$
\vec{\phi}^{k+1}=\left(1-\lambda_{k}\right) \vec{\phi}^{k}+\lambda_{k} \vec{y}^{k}
$$

5. If Eq. (10) is satisfied, stop the iteration; if not, return to step 2.

\section{Numerical experiments}

In this section, we provide a numerical example to demonstrate the effectiveness of the model and the numerical algorithm. A polycentric urban city (see Figure 4) with two CBDs is considered. The city spans about $37 \mathrm{~km}$ from west $(x \approx 4 \mathrm{~km})$ to east $(x \approx$ $41 \mathrm{~km})$ and $24 \mathrm{~km}$ from south $(y \approx 10 \mathrm{~km})$ to north $(y \approx 34 \mathrm{~km})$. CBD 1 and CBD 2 are located at $(14 \mathrm{~km}, 20 \mathrm{~km})$ and $(31 \mathrm{~km}, 23 \mathrm{~km})$, respectively, and the radiuses of both are $1 \mathrm{~km}$. Denote the travelers to CBD 1 and CBD 2 by Class 1 and Class 2, respectively. The modeling period is 5 hours, i.e., $T=[0 \mathrm{hr}, 5 \mathrm{hr}], t_{\text {end }}=5 \mathrm{hr}$. The elastic demand, which is associated with the total travel cost, is set to be

$$
q^{m}\left(\phi^{m}(x, y, t), t\right)=q_{m a x}^{m}\left[1-\gamma_{1} \phi^{m}(x, y, t)\right] g^{m}(t), \quad m=1,2,
$$

where $q_{\text {max }}^{m}$ (in $v e h / \mathrm{km}^{2} / \mathrm{hr}$ ) is the maximum of Class $m$ and $\gamma_{1}=0.002 \$^{-1}$. The factor $1-\gamma_{1} \phi^{m}(x, y, t)$ means that more travelers are generated in the area with lower cost. The function $g^{m}(t)(m=1,2)$ represents the time-dependent natural demand. We define

$$
g^{1}(t)= \begin{cases}t / 2, & t \in[0 h r, 2 h r], \\ 2-t / 2, & t \in[2 h r, 4 h r], \\ 0, & t \in[4 h r, 5 h r],\end{cases}
$$




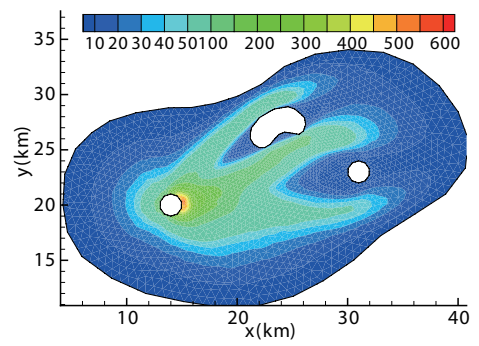

(a) $t=1 \mathrm{hr}$

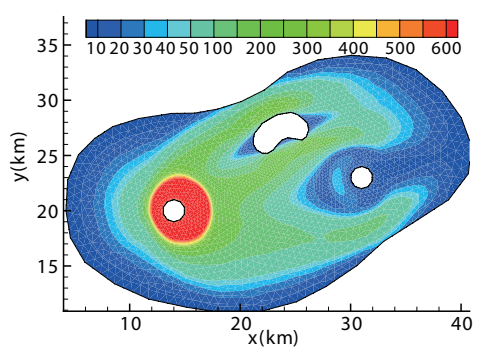

(c) $t=2 \mathrm{hr}$

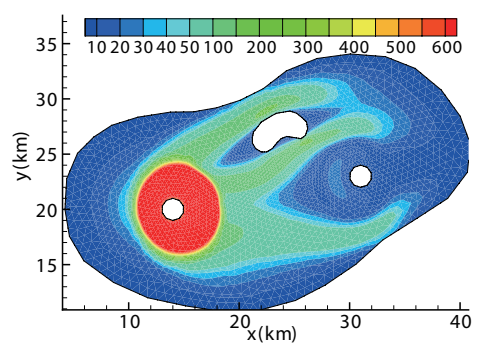

(e) $t=3 \mathrm{hr}$

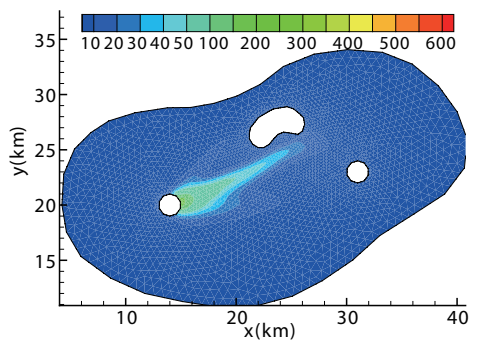

(g) $t=4 h r$

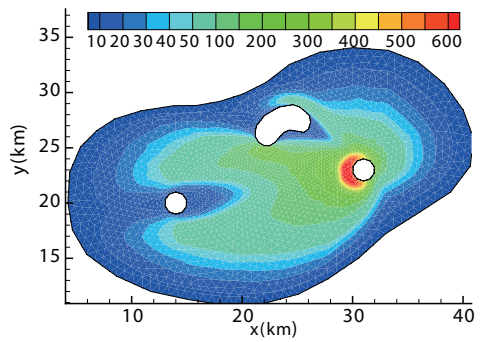

(b) $t=1 \mathrm{hr}$

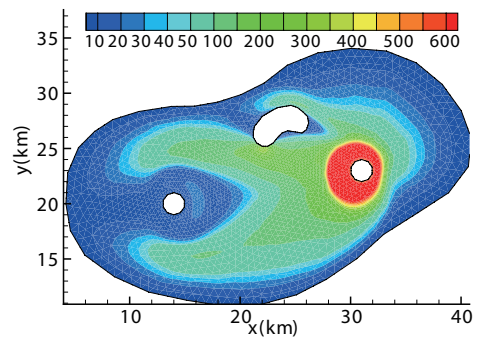

(d) $t=2 \mathrm{hr}$

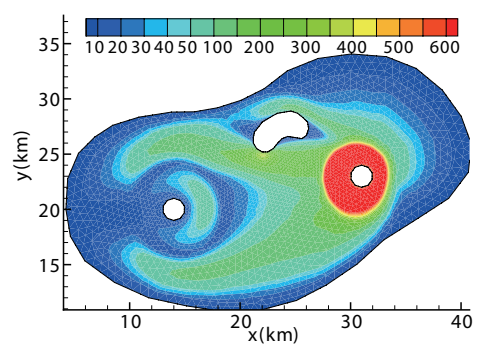

(f) $t=3 \mathrm{hr}$

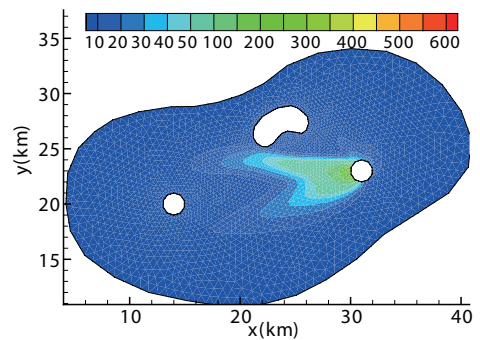

(h) $t=4 \mathrm{hr}$

Figure 8. Density plot (left: Class 1; right: Class 2).

and

$$
g^{2}(t)= \begin{cases}t, & t \in[0 \mathrm{hr}, 1 \mathrm{hr}], \\ 1, & t \in[1 \mathrm{hr}, 3 \mathrm{hr}], \\ 4-t, & t \in[3 \mathrm{hr}, 4 \mathrm{hr}], \\ 0, & t \in[4 \mathrm{hr}, 5 \mathrm{hr}] .\end{cases}
$$




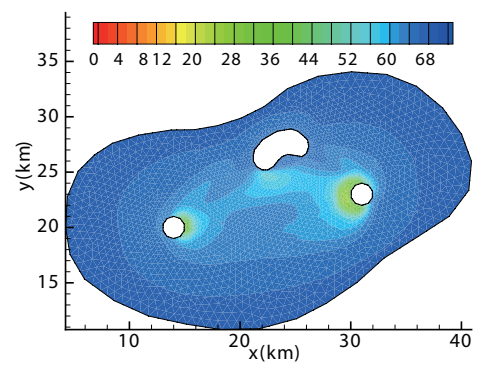

(a) $t=1 \mathrm{hr}$

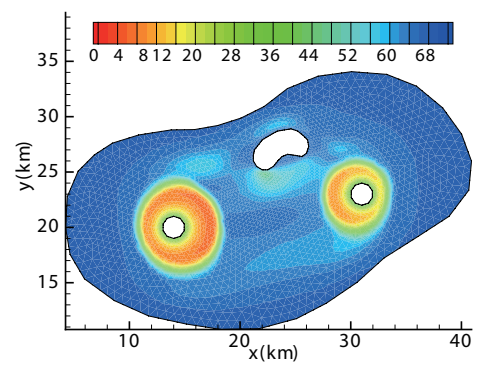

(c) $t=3 \mathrm{hr}$

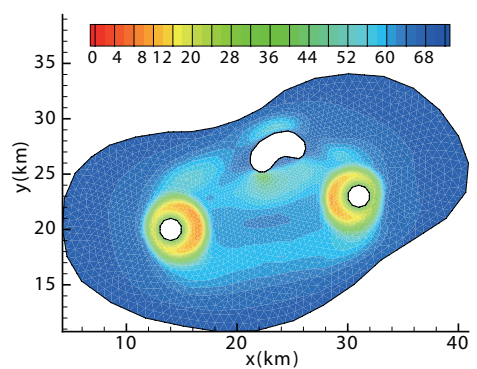

(b) $t=2 h r$

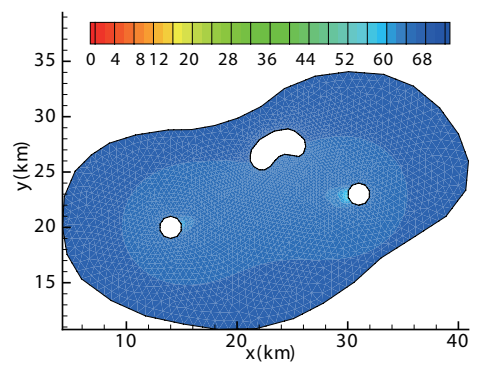

(d) $t=4 h r$

Figure 9. Speed plot.

In Eq. (1), we set the parameter $\beta=2 \times 10^{-6}$, and the free-flow speed is

$$
U_{f}^{m}(x, y)=U_{\max }^{m}\left[1+\gamma_{2} \frac{d^{1}(x, y)}{d_{\max }^{1}} \frac{d^{2}(x, y)}{d_{\max }^{2}}\right],
$$

where $U_{m a x}^{m}$ is the maximum speed of the Class $m, d^{m}(x, y)$ is the distance between $(x, y)$ and the center of the $m$-th CBD, $d_{\max }^{m}=\max _{(x, y) \in \Omega} d^{m}(x, y)(m=1,2)$ and $\gamma_{2}=0.12 \mathrm{~km}^{-1}$. Then, we can compute the critical density is $353.55 \mathrm{veh} / \mathrm{km}^{2}$. We set $U_{\max }^{1}=U_{\max }^{2}=65 \mathrm{~km} / \mathrm{hr}$ and compute $d_{\max }^{1}, d_{\max }^{2}$ are $27.6 \mathrm{~km}$ and $26.9 \mathrm{~km}$, respectively. In Eq. (2), we set $\kappa=75 \$ / h r$ and

$$
\left\{\begin{array}{l}
\pi^{1}(\tilde{\rho}(x, y, t))=\pi_{0}\left[\frac{\rho^{2}(x, y, t)}{\rho^{1}(x, y, t)+\rho^{2}(x, y, t)}\right]^{2}+\pi_{1}\left(\rho^{1}(x, y, t)+\rho^{2}(x, y, t)\right)^{2}, \\
\pi^{2}(\tilde{\rho}(x, y, t))=\pi_{0}\left[\frac{\rho^{1}(x, y, t)}{\rho^{1}(x, y, t)+\rho^{2}(x, y, t)}\right]^{2}+\pi_{1}\left(\rho^{1}(x, y, t)+\rho^{2}(x, y, t)\right)^{2},
\end{array}\right.
$$

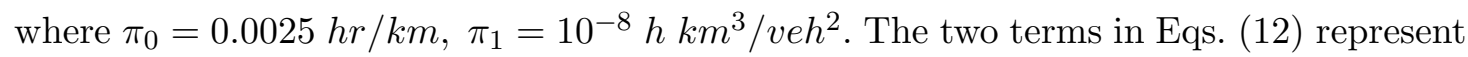
the preference for avoiding conflict with the other class of traffic flow and the preference for avoiding the high-density region, respectively.

We assume that there is no traveler at the beginning of the modeling period and no cost incurred by entering each CBD. Thus, we have $\rho_{0}^{m}(x, y)=0, \forall(x, y) \in \Omega(m=1, \ldots, M)$ and $\phi^{m}(x, y, t)=0, \forall(x, y) \in \Gamma_{C B D}^{m}, t \in T(m=1, \ldots, M)$. We use three meshes (Mesh 1: 1457 nodes, 2756 elements and $N_{t}=3000$; Mesh 2: 3178 nodes, 6107 elements and $N_{t}=6000 ;$ Mesh 3: 4998 nodes, 9693 elements and $\left.N_{t}=6000\right)$ in the numerical example.

Let us consider the convergence and effectiveness of the self-adaptive MSA in which Mesh 2 is considered. Figure 5 compares the step size between the self-adaptive MSA and the conventional MSA. We can see that the step size of the self-adaptive MSA is 


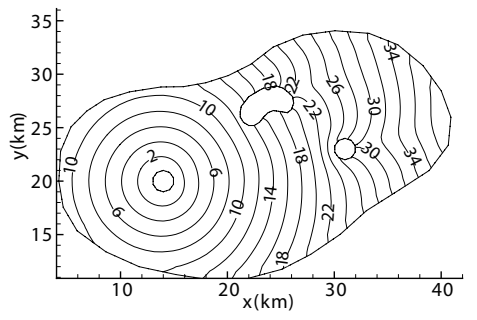

(a) $t=1 \mathrm{hr}$

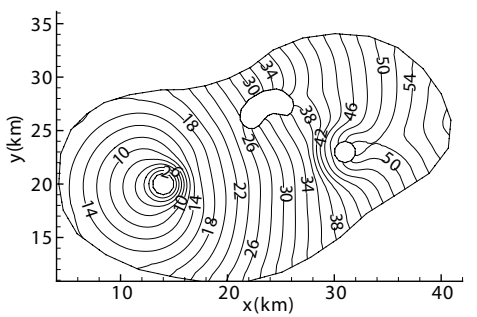

(c) $t=2 \mathrm{hr}$

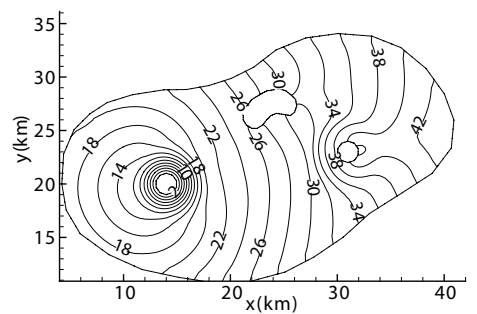

(e) $t=3 \mathrm{hr}$

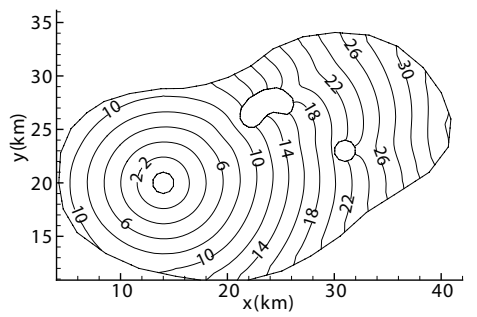

(g) $t=4 h r$

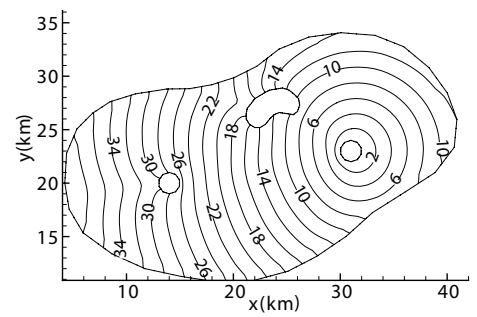

(b) $t=1 \mathrm{hr}$

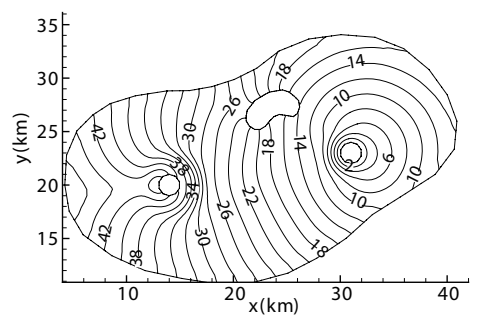

(d) $t=2 h r$

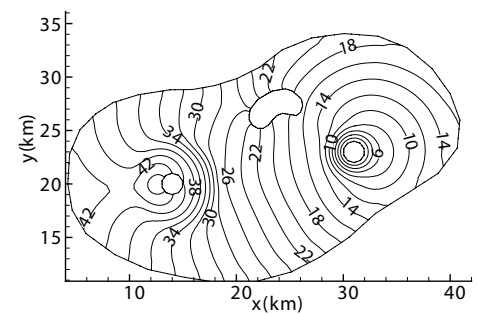

(f) $t=3 \mathrm{hr}$

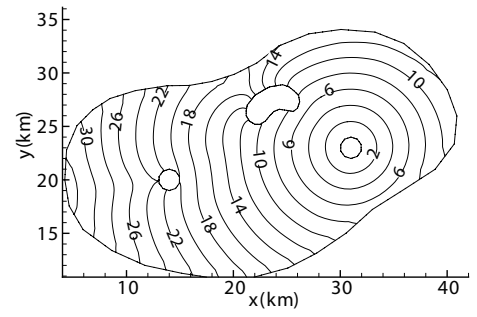

(h) $t=4 \mathrm{hr}$

Figure 10. Cost potential plot (left: Class 1; right: Class 2).

Table 1. Number of iterations of the conventional MSA and self-adaptive MSA.

\begin{tabular}{lcc}
\hline & Conventional MSA & Self-adaptive MSA \\
\hline Mesh 1 & 261 & 48 \\
Mesh 2 & 499 & 121 \\
Mesh 3 & 706 & 128 \\
\hline
\end{tabular}

almost constant after several steps, which can be viewed as the optimal step size, and is much larger than that of the conventional MSA. Figure 6 illustrates the errors of the self-adaptive MSA and the conventional MSA. From the figure, we can see convergence of the self-adaptive MSA. Table 1 shows the number of iterations of the two methods with different meshes. We can see that the self-adaptive MSA method is more efficient than the conventional MSA. 


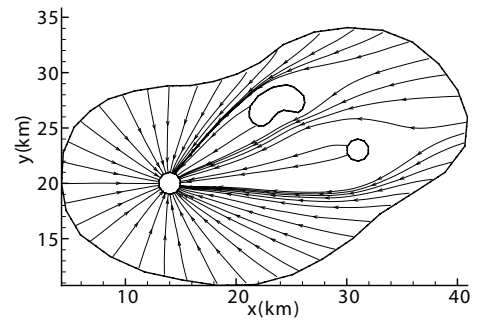

(a) $t=1 \mathrm{hr}$

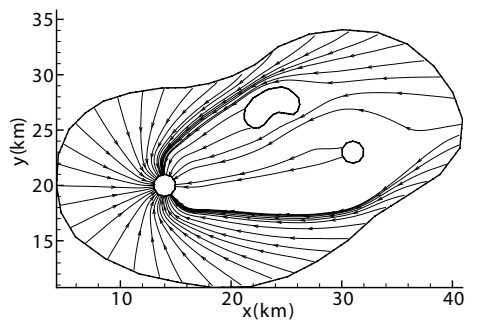

(c) $t=2 h r$

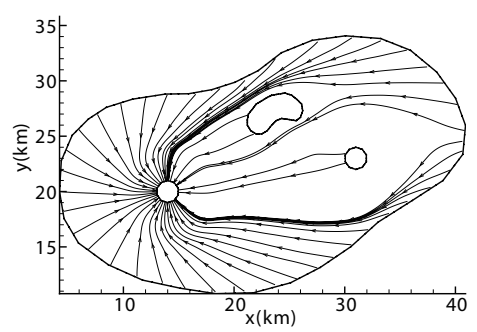

(e) $t=3 \mathrm{hr}$

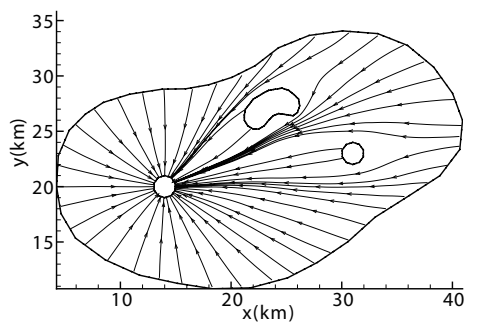

(g) $t=4 h r$

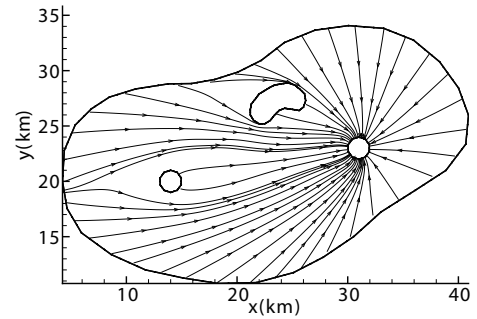

(b) $t=1 \mathrm{hr}$

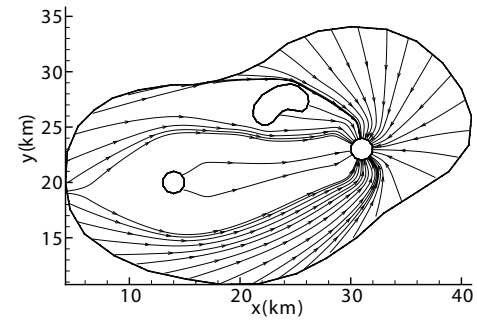

(d) $t=2 h r$

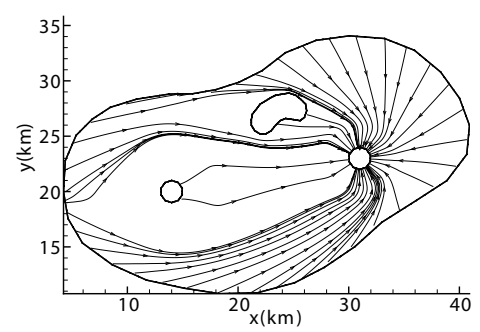

(f) $t=3 \mathrm{hr}$

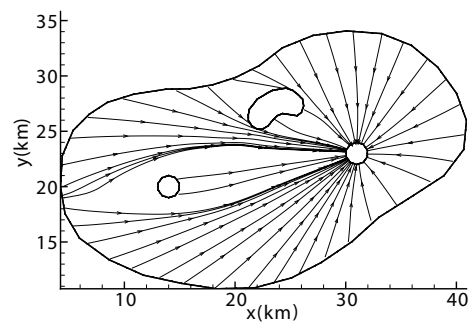

(h) $t=4 h r$

Figure 11. Flow vector plot (left: Class 1; right: Class 2).

We now consider the convergence of different meshes. The density of Class 1 along the Line $1\left(y=\frac{3}{4}(x-14)+20\right)$ and the density of Class 2 along the Line $2\left(y=-\frac{1}{3}(x-31)+23\right)$ are shown in Figure 7 . We can see good convergence of the meshes. Mesh 2 is adopted for further discussion.

Figures 8 and 9 show the density and speed distributions of Class 1 and Class 2, respectively. At $t=1 \mathrm{hr}$, Class 1 is in the free-flow state and the density near CBD 2 is very low (Figure $8(\mathrm{a})$ ), whereas Class 2 exceeds the critical density at the western boundary of the CBD 2 and the density near CBD 1 is very low (Figure 8(b)). As the demands increase, the two classes of traffic flow are both in a congestion condition around each CBD at $t=2 h r$ (Figure 9(b)). The density of Class 1 is higher in the eastern region 


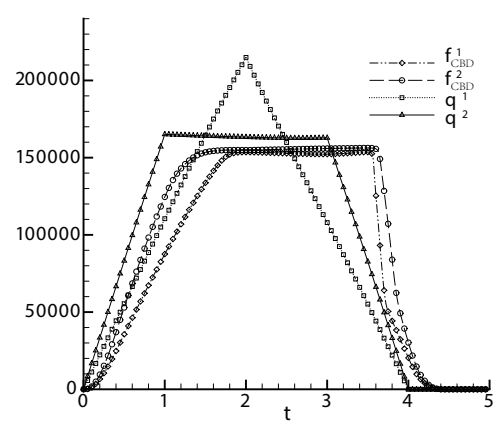

(a)

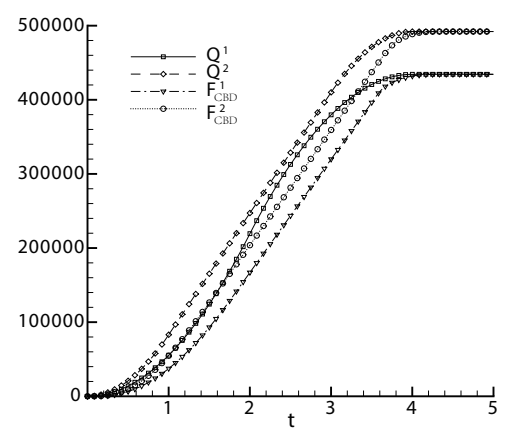

(b)

Figure 12. (a) The total demand and total inflow; (b) The cumulative demand and cumulative inflow.

of CBD 1 because there are more travelers arriving at CBD 1 from the east of the city (Figure 8(c)). Similarly, the density of Class 2 is higher in the western region of CBD 2 (Figure 8(d)). At $t=3 \mathrm{hr}$, although the demand of Class 1 is lower, the regions around the CBD 1 and CBD 2 are still in the congestion state due to the limitation on the traffic inflow into the CBDs (Figure 8(e), (f) and Figure 9(c)). As the demands continuously decrease, the two classes of traffic flow both revert to the free-flow state at $t=4 \mathrm{hr}$ (Figure 8(g), (h) and Figure 9(d)).

Figure 10 illustrates the contours of the cost potential of Class 1 and Class 2 at different times. At the beginning of the modeling period, when the density of Class 1 is low and the traffic is in a free-flow state, the cost potential contours are a series of concentric circles located around CBD 1 (Figure 10(a)). The cost potential contours of Class 2 on the western side of CBD 2 are slightly denser than those on the eastern side (Figure 10(b)). At $t=2 \mathrm{hr}$ and $t=3 \mathrm{hr}$, the contours become denser, especially in the region around the CBDs, due to the high traffic density (Figure 10(c)-(f)), which means that the local cost increases. As the traffic demands decrease, the traffic flow reverts to the free-flow state, and the cost potential contours of the two classes again become concentric circles (Figure 10(g), (h)).

Figure 11 shows the temporal and spatial distributions of the flow vector of Class 1 and Class 2, which reflect the route-choice strategy. Travelers bypass the lake and the other CBD. At the beginning and the end of the modeling period, the density is low and travelers almost move along straight lines pointing to the center of the CBD (Figure 11(a), (b), (g), (h)). As the density in the eastern area around CBD 1 and the western area of CBD 2 increases, the travelers choose curved routes to avoid the area (Figure 11(c), (d), (e), (f)).

The total demand of the Class $m(\mathrm{~m}=1,2)$ over the whole domain at time $t$ is

$$
q^{m}(t)=\iint_{\Omega} q^{m}\left(\phi^{m}(x, y, t), t\right) d x d y
$$

and the cumulative demand is

$$
Q^{m}(t)=\int_{0}^{t} q^{m}(\xi) d \xi
$$


The total inflow of Class $m$ through $\Gamma_{C B D}^{m}$ is

$$
f_{C B D}^{m}=\oint_{\Gamma_{C B D}^{m}}\left(\boldsymbol{F}^{m} \cdot \boldsymbol{n}\right)(x, y, t) d s .
$$

and the cumulative inflow is

$$
F_{C B D}^{m}=\int_{0}^{t} f_{C B D}^{m}(\xi) d \xi
$$

The total demands, inflows, the corresponding cumulative demands and the corresponding cumulative inflows of the two classes of traffic flow are shown in Figure 12. At the beginning of the period, the total demands and inflows of each class are both increased due to the increasing traffic demand. After about $t=1.6 \mathrm{hr}$, the total inflows of the two classes reach the maximum value and no longer increase because the density around the CBDs is greater than the critical density $\rho_{c}$, and the capacities of the two CBDs are very similar. After about $t=3.7 h r$, traffic flow returns to the non-congestion state, and the inflows of each class begin to fall. The inflows decrease to 0 after about $t=4.3 \mathrm{hr}$ (Figure 12(a)). For each class, the corresponding cumulative inflow $Q_{C B D}^{m}$ is always lower than the corresponding cumulative demand $Q^{m}(t)$, which describes a traffic delay. However, the two curves finally coincide, which implies that all travelers have reached the CBDs.

\section{Conclusions}

A PDUO-C model is extended to study the traffic equilibrium problem for a polycentric urban city with an arbitrary configuration. The model satisfies the predictive dynamic user-optimal principle, which describes the route choice behavior of each class of traffic flow. The model consists of two interconnected parts, the conservation law part and the Hamilton-Jacobi part, with different initial times. Each class of the traffic flow follows its own conservation law and Hamilton-Jacobi equation. However, different classes are not independent and the interactions are described by the cost function. We regard it as a fixed-point problem and use a self-adaptive MSA, which can automatically determine the optimal MSA step size, to solve the problem. The finite volume method is applied to solve the conservation equations and the Hamilton-Jacobi equations on unstructured meshes. The numerical results show that the model can describe the macroscopic dynamic characteristics of the traffic flow for a polycentric city, and the numerical algorithm is effective.

In this paper, we consider that the density of each class of traffic flow is a function of the total density. It would be reasonable to consider the influence of the directions of other classes in future work.

\section{Acknowledgements}

This work was jointly supported by grants from the Research Grants Council of the Hong Kong Special Administrative Region, China (No. 17208614), the National Natural Science Foundation of China (No. 11272199, 11202175), the National Basic Research Program of China (2012CB725404), the Shanghai Program for Innovative Research Team in Universities, and the National Research Foundation of Korea (NRF) grant funded by the Korea government (MSIP) (NRF-2010-0029446). 


\section{References}

Abgrall, R. 1996. "Numerical discretization of the first-order Hamilton-Jacobi equation on triangular meshes." Communications on pure and applied mathematics 49 (12): 1339-1373.

Blumenfeld, D. E. 1977. "Modeling the joint distribution of home and workplace locations in a city." Transportation Science 11 (2): 307-337.

Boyce, D. E., Ran, B., and Leblanc, L. J. 1995. "Solving an instantaneous dynamic user-optimal route choice model." Transportation Science 29 (2): 128-142.

Buckley, D. J. 1979. "Traffic assignment in a two-dimensional continuous representation of a traffic network with flow-dependent speeds." Transportation Research Part B: Methodological 13 (2): 167-179.

Chow, A. H. F. 2009. "Dynamic system optimal traffic assignment-a state-dependent control theoretic approach." Transportmetrica 5 (2): 85-106.

Du, J., Wong, S. C., Shu, C. W., Xiong, T., Zhang, M. P., and Choi, K. 2013. "Revisiting Jiangs dynamic continuum model for urban cities." Transportation Research Part B: Methodological 56: $96-119$.

Du, Y. C., Wong, S. C., and Sun, L. J. 2016. "A multi-commodity discrete/continuum model for a traffic equilibrium system." Transportmetrica A: Transport Science 12: 249-271.

Ho, H. W., and Wong, S. C. 2007. "Housing allocation problem in a continuum transportation system." Transportmetrica 3 (1): 21-39.

Ho, H. W., Wong, S. C., and Sumalee, A. 2013. "A congestion-pricing problem with a polycentric region and multi-class users: a continuum modelling approach." Transportmetrica A: Transport Science 9 (6): 514-545.

Hoogendoorn, S. P., and Bovy, P. H. L. 2004. "Dynamic user-optimal assignment in continuous time and space." Transportation Research Part B: Methodological 38 (7): 571-592.

Huang, L., Wong, S. C., Zhang, M. P., Shu, C. W., and Lam, W. H. K. 2009. "Revisiting Hughes' dynamic continuum model for pedestrian flow and the development of an efficient solution algorithm." Transportation Research Part B: Methodological 43 (1): 127-141.

Hughes, R. L. 2002. "A continuum theory for the flow of pedestrians." Transportation Research Part B: Methodological 36 (6): 507-535.

Jiang, Y. Q., Wong, S. C., Ho, H. W., Zhang, P., Liu, R. X., and Sumalee, A. 2011. "A dynamic traffic assignment model for a continuum transportation system." Transportation Research Part B: Methodological 45 (2): 343-363.

Jiang, Y. Q., Wong, S. C., Zhang, P. and Choi, K. 2014. "Dynamic continuum model with elastic demand for a polycentric urban city." Transportation Science, in press.

Jiang, Y. Q., Xiong, T., Wong, S. C., Shu, C. W., Zhang, M. P., Zhang, P., and Lam, W. H. K. 2009. "A reactive dynamic continuum user equilibrium model for bi-directional pedestrian flows." Acta Mathematica Scientia 29 (6): 1541-1555.

Kuwahara, M., and Akamatsu, T. 2001. "Dynamic user optimal assignment with physical queues for a many-to-many OD pattern." Transportation Research Part B: Methodological 35 (5): 461-479.

Lo, H. K., and Szeto, W. Y. 2002. "A cell-based variational inequality formulation of the dynamic user optimal assignment problem." Transportation Research Part B: Methodological 36 (5): 421-443.

Nie, Y. M. 2011. "A cell-based Merchant-Nemhauser model for the system optimum dynamic traffic assignment problem." Transportation Research Part B: Methodological 45 (2): 329-342.

Robbins, H., and Monro, S. 1951. "A stochastic approximation method." The Annals of Mathematical Statistics 22 (3): 400-407.

Sheffi, Y. 1984. "Urban transportation networks: Equilibrium analysis with mathematical programming techniques." Prentice Hall, New York.

Szeto, W. Y., and Lo, H. K. 2004. "A cell-based simultaneous route and departure time choice model with elastic demand." Transportation Research Part B: Methodological 38 (7): 593-612.

Taguchi, A., and Iri, M. 1982. "Continuum approximation to dense networks and its application to the analysis of urban road networks." Applications. Springer, Berlin Heidelberg. 178-217

Tao, Y. Z., Jiang, Y. Q., Du, J., Wong, S. C., Zhang, P., Xia, Y. H., and Choi, K. 2014. "Dynamic 
system-optimal traffic assignment for a city using the continuum modeling approach." Journal of Advanced Transportation 48 (7): 782-797.

Tong, C. O., and Wong, S. C. 2000. "A predictive dynamic traffic assignment model in congested capacity-constrained road networks." Transportation Research Part B: Methodological 34 (8): 625-644.

Vaughan, R. 1987. "Urban spatial traffic patterns." Pion Ltd., London.

Wong, S. C. 1998. "Multi-commodity traffic assignment by continuum approximation of network flow with variable demand." Transportation Research Part B: Methodological 32 (8): 567-581.

Wong, S. C., Lee, C. K., and Tong, C. O. 1998. "Finite element solution for the continuum traffic equilibrium problems." International Journal for Numerical Methods in Engineering 43 (7): 1253-1273.

Wong, W., and Wong, S. C. 2015. "Systematic bias in transport model calibration arising from the variability of linear data projection." Transportation Research Part B: Methodological 75: 1-18.

Wong, W., and Wong, S. C. 2016. "Network topological effects on the macroscopic Bureau of Public Roads function." Transportmetrica A: Transport Science 12: 272-296.

Yin, J., Wong, S. C., Sze, N. N., and Ho, H. W. 2013. "A continuum model for housing allocation and transportation emission problems in a polycentric city." International Journal of Sustainable Transportation 7 (4): 275-298. 